\title{
Analogies between Antibodies and Simpler Chemical Substances
}

\author{
LINUS PAULING, Director, Gates and Crellin Laboratories, California \\ Institute of Tecinnology, Pasadena, Calif.
}

Because of his contributions in the study of antibodies, Linus Pauling was chosen as the first Harrison Howe lecturer. His talk, given below, was delivered before the Rochester Section of the AMr erican Chemicat. Society, Feb. 4, in Rochester, N. Y.

LET ME express my deep appreciation of the honor of presenting the first Harrison Howe Menorial Lecture, and my thanks to you for selecting me. I am sure that Harrison Howe, with his broad interests, covering the whole of chemistry and technology, would not consider out of place the subject which I have chosen: "Analogies between Antibodies and Simpler Chemical Substances".

I became interested in antibodies ten years ago. Dr. Karl Landsteiner asked me to come to his laboratory at the Rockefeller Institute for Medical Research-he knew that I had been working on the magnetic properties and structure of hemoglobin, and that I was interested in the grent geperal problem of the structure of proteins-and when.I went to see him he asked me many questions about the possible interpretation of serological phenomena in terms of the detailed structure of molecules. Having little knowledge of serology, I found it necessary to ask him many questions before I could attempt to answer his; and he was good enough to permit me to spend a day with him on each of several later trips which $I$ made to New York; and then, in December of 1937, when I was at Cornell as George Fisher Baker Lecturer in Chemistry, he came to Ithaca for a visit of several days, which we devoted to the intensive and critical discussion of immunological experiments and their interpretation. This was a great experience for me--to have this field of knowledge presented and clarified to me by its great Master, the man who had contributed more to it and had thought more deeply about it than any other living being; and I have a deep feeling of gratitude to him for his kindness and interest. Dr. Landsteiner, as well as Profassor Michael Heidelberger, encouraged me, in 1940 , to publish my first paper on the structure of antibodies and to begin experimental work in this field, and he followed the course of the work, carried on in Pasadena in collaboration with Dan H. Campbell, David Pressman, and several graduate students, and with the support of The Rockefeller Foundation, until his death in 1943.

\section{Antibodies as Chemical Substances}

Chemists are not accustomed to thinking of antibodies as chemical substances, nor of the serological reactions shown by antibodies as chemical reactions. It is true that antibodies, contrining tens of thousands of atoms in each molecule, are far more complex than simple chemical substances, with around 10 or 20 atoms per molecule; and some immunological reactions, such as the anaphylactic shock shown by a previously sensitized animal when it receives a small injection of the antigenic substance to which it is sensitive, do not have much in common with chemical reactions. Nevertbeless, a comparison of antibodies with simple chemical substances and of serological reactions with ordinary chemical reactions provides a basis for understanding antibodies and their behavior, and also suggests new experiments to be carried out.

When an antigen (a substance, usually a protein, foreign to the animal) is injected into an animal, it may cause the production in the blood stream and cells of the animal of substances which are related in their properties to the antigen which was injected. These substances are called antibodies.

Antibodies are proteins (serum globulin), with very large molecules, the molecular weight being often 160,000 and sometimes as great as $1,000,000$. A striking

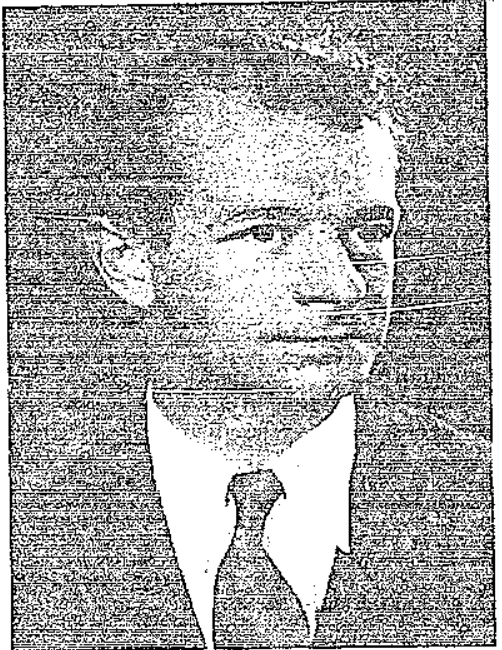

property possessed by most antibodiez and antitoxins is that of forming a precipitate with the corresponding antigen. For example, when an animal is injected with egg albumin, antibodies homologous to the egg albumin are formed, and the serum of this animal gives a precipitate when it is mixed with a solution of egg albumin, the precipitate containing both antibody and egg albumin. A most striking property of antibodies is their specificity - the serum homologous to egg albumin may give a very large precipitate when mixed with a solution of egg albumin, and give no precipitate at all when mixed with a solution of any other protein. There are some exceptions to this specificity: An antiserum homologous to hen egg albumin will give a precipitate with duck egg albumin, but the quantity of the precipitate will be smaller than that formed with hen egg albumin. This specificity is almost limitless in its extent. It was shown by Landsteiner that antisera can be produced which react with different chemical groupings attached artificially to proteins, and these chemical groups can be synthesized in number almost without limit.

The two striking properties of antibodies-the formation of a precipitate, and the great specificity of the reaction between antibody and antigen-have very interesting analogs in the chemistry of simple chemical substances.

\section{Serologicai Precipitation}

After several decades during which the nature of serological precipitation was in doubt, it has now been shown by convincing experiments that 8 serological precipitate is a framework of antigen and antibody molecules; with molecules of both antigen and antibody in the framework attached to their neighbors by' two or more bonds. The molecules of antigen and of antibody are thus to be considered as having a valence (analogous to, but not identical with, ordinary chemical valence) 
equal to or greater than 2. There is some evidence that in most antisera many of the antibody moleoules have two combining regions - that is, they are bivalert. Some antisera seem to contain univalent antibodies, capable of combining is $\therefore$ h ant' yen molecules, but not of forming a precupitate with them. The mechanism of the forroation of a serological preeipitate is the following: Each antigen molecule attaches to itself two antigen molecules, this process continuing until the framework of molecules reaches macroscopic size, constituting a precipitate. Many of the ordinary precipitates that the chemist meets in his work are formed in the same way. An exsmple is the precipitate of silver cyanide produced when a solution of a silver salt is mixed with a solution containing cysnide ion. The silver ion has the property of forming two covalent bonds with cyanide jon; it attaches to itself two cyanide ions, which stick out on either side of it. The cyanide ion also has the property of forming two covalent bonds, one formed by the carbon atom and one by the nitrogen atom. In this way long chains of altemating bicovalent silver and bicovalent cyanide groups are formed, and ultimately these long chains arrange themselves side by side to form the silver cyanide precipitate. A similar process is involved in the precipitation of Prussian blue when a solution containing ferrous ion is mixed with a solution containing ferricyanide ion. The ferricyanide ion contains six groups attached to a central ferric atom. Each of these six groups is capable of attaching itself to a ferrous ion, and the ferrous ion can form bonds with six cyanide groups. This process leads to the formstion of a cubical framework extending through epace, the framework of the Prussian blue precipitate.

It is, moreover, of interest that the serological precipitate can be redissolved by addition of an excess of antigen, just as can the silver cyanide precipitate by an $\mathrm{ex}-$ cess of cyanide ion; in each system the effect is due to the formation of soluble complexes.

\section{Specificity of Anfibodies}

The second property of antibodies which has an analog in the chemistry of simple substances is the great specificity of interaction of antibody and the homologous antigen. It has been shown that this specificity is due to a striking complementariness in structure between a portion of the surface of the antigen molecule and a combining region on the antibody. After the antigen is injected into the animal, antibody molecules are synthesized by the animal in such a way that a region (two regions for a bivalent antibody) of the antibody assumes a configuration which mirrors a portion of the surface of the antigen molecule. This complementariness in structure leads to a strong atiraction between the sntibody molecule and the antigen, becsuse it permits this combining region of the antibody molecule to get into close contact with the antigen molecule; the closer that two molerules can gett in contact with one another, the stronger the intermolecular force of attraction between thern. A protein molecule serving as antigen usually has a rather complicated surface structure, and it is easy.to see how the great specificity shown by antibodies arises. The reaction shown by simple chemical substances which is analogous to that of specific combination of antigen and antibody is the formation of a crystal of a substance from solution. A crystal of a molecular substance is stable because all of the molecules pile themselves into such a configuration that each molecule is surrounded as closely as possible by other molecules-that is, if a molecule were to be removed from the interior of a crystal, the cavity that it would leave would have very nearly the shape of the molecule itself. We can say that the part of a crystal other than a given molecule is very closely complementary to that molecule. Other molecules, with different shape and structure, would not fit into this cavity nearly so well, and in consequence other molecules in general would not be incorporated in a growing crystal. This is the explanation of the astounding chemical process of purification by crystallization-from a very complicated system, such as, for example, grape jelly, containing hundreds of different kinds of molecules, crystals which are nearly chemically pure may be formed, such as crystals of cream of tartar, potassium hydrogen tartrate.

Deviation from specificity occurs in serological reaction when an antiserum combines with an antigen other than the immunizing antigen, and it occurs in crystallization when solid solutions are formed. There are striking similarities between these two kinds of deviation. For example, the antibody to an azoprotein containing azobenzoic acid with a bromine atom in the meta position shows strong precipitation with an azoprotein containing azobenzoic acid with a methyl group in the meta position; this complete cross reaction is the result of the fact that the bromine atom and the methyl group have very nearly the same shape and size, although their.chemical properties otherwise are quite different. Similarly, solid solutions are formed by corresponding bromine-substituted and methyl-substituted benzenes.

I could give many more examples in illustration of my thesis, but I shall refrain, remembering the observation by Voltaire that "the secret of being a bore is to tell everything".

\section{Potentialities of the Theory in.Other Fields}

Although crystallization is the only simple chemical reaction which shows striking similarity to serological reactions with respect to specificity, there are many physiological phenomena which are sim- ilarly specific, and for which the specificity can be given a similar explanation. The specificity of the catalytic activity of enzymes is duc to a surface configuration of the enzyme such as to make the enzyme complementary to the substrate molecule, or, rather, to the subst rate molecule in the strained state that occurs during the cat talyzed reaction. The specific action of drugs and bactericidal substances has a similar explanation. Even the senses of taste and odor are based upor molecular configuration rather than upon ordinary chemical properties-a molecule which has the same shape as a camphor molecule will smell like camphor even though it may be quite unrelated to camphor chemically. I am convinced that it will be found in the future, as our understanding of pliysiological phenomena becomes deeper, that the shapes and sizes of molecules are of just as great significance in determining their physiological behavior as are their internal structure and ordinary chemical properties. I believe that the thorough invegtigation of the shapes and sizes of molecules will lead to great advances in fundamental biology and medicine; and because of this belief I am now turning my efforts in this directicn. It seems clear that complementariness of the sort we have been considering must be involved in the autocatalytic activity (production of replicas) and heterocatalytic activity (production of other specific molecules) of the tens of thousands of genes that carry to us our inheritance from our ancestors. And, moreover, I am convinced that progress in our attack against disease, in our better understanding of the human body, of bacteria, viruses, and other vectors of disease, and of their interaction with each other depends on a better understanding of intermolecular forces and inteructions. "The long habit of living indisposeth us for dying"; and, as we have got better and better control over the infectious diseases, the degenerative diseases-cuncer, cardiovascular disease - hrve become more and more important, and call more and more urgently for control: but we cumnot yet attack these diseases in a straightforward way-we need leads, hints, which a better understanding of the phenomene of growth, development, and disease in terme of molecular structure would surely provide. I have hope, that it will be possible to carry on a program of research in molecular structure that will lead to significant contributions to biology and to medicine.

Nearly sixty years ago wait Winitman wrote about Life and Death:

The two old, simple problems ever intertwined,

Close home, elusive, present, baffled, grappled,

By each successive age insoluble, pussed on To ours today - and we pass on the same.

Let us resolve that as we pass on these problems to our following generation we shall make also our contribution toward their solution. 